\title{
Tolérer les non-fumeurs?
}

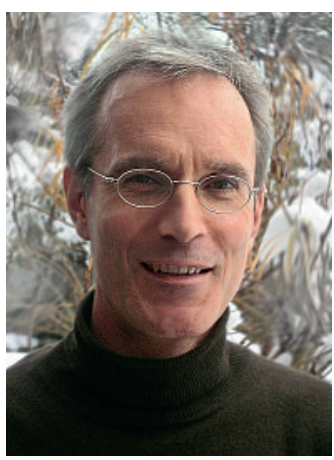

Bruno Kesseli
L'un des avantages de cette période de tranquillité entre Noël et Nouvel An réside dans le fait qu'on y trouve plus facilement le temps de réanimer - sans la moindre connotation médicale - de vieilles amitiés. Ce retour dans mon propre passé s'annonçait d'autant plus intense, qu'avec ce vieil ami nous avions convenu d'aller manger dans le bistrot que nous avions l'habitude de fréquenter à l'époque. Pour celui qui n'avait plus mis les pieds dans ce lieu depuis les plus ou moins glorieuses années 80 , l'expérience fut d'une grande intensité atmosphérique. Pénétrer dans l'établissement revenait à se sentir plaqué contre un mur de fumée, avec un impact qui rappelait l'effet d'un crochet habilement décroché par un boxeur poids lourd. En d'autres termes, on suffoque et pense immédiatement à la réanimation, au sens médical du terme cette fois.

J'avoue que je force un peu le trait, mais je me demande néanmoins comment, il y a 25 ans de cela, j'étais en mesure de supporter avec une belle régularité cette atmosphère saturée de poussières fines. Le sujet de discussion était tout trouvé, car mon ami est non seulement spécialiste en sciences humaines et journaliste culturel, mais surtout - le contexte s'y prête - fumeur. Il en a résulté un débat animé, à l'instar des centaines d'autres sur le même sujet. Comme souvent, lorsqu'on avance des arguments médicaux probants et quasiment irréfutables, les réponses sont d'ordre philosophique: liberté de l'individu et ses limites, responsabilité individuelle et mise en danger de tiers, plaisir et risque.

Les esprits des grands penseurs de Locke à Kant en passant par Luhmann et d'autres sont convoqués pour étayer les arguments de l'un ou de l'autre dans

\section{Le fumeur tolérant permet aux autres de manger à sa table}

ce lieu où la fumée était telle qu'elle nous avait déjà, à bien des égards, passablement fait perdre toute vue d'ensemble. Mais il est vrai que nous sommes venus délibérément ici (contrairement aux employés).

La sophistique nous avait-elle fatigué ou voulions-nous trouver une issue favorable à la discussion, le fait est que nous en sommes arrivés à parler de tolérance. D'après mon ami, si les deux parties (fumeurs et non-fumeurs) faisaient preuve de suffisamment de tolérance, cela résoudrait bien des problèmes. Sans citer Voltaire ou Lessing cette fois, le voilà qui devenait concret et pratique. Et il ajouta d'un ton convaincu que d'après son expérience, les fumeurs sont très tolérants en général. Pour lui, comme pour bon nombre d'autres personnes, il est en effet normal d'attendre que les autres convives aient fini de manger avant d'allumer une cigarette.

Peux-tu répéter? Ai-je bien entendu? Mon ami a pourtant de solides connaissances linguistiques. Nous sommes néanmoins d'accord que tolérance vient de «tolerare» qui signifie entre autres «autoriser», «permettre», «supporter». En permettant aux autres personnes qui se tiennent à sa table d'adopter un comportement (manger en l'occurrence) nuisant gravement à son bien-être, le fumeur ferait donc preuve de tolérance? Quel mérite. Par analogie, on pourrait donc dire aussi que la femme enceinte qui renonce à fumer pendant sa grossesse fait preuve d'une grande tolérance envers les exigences de l'enfant qu'elle porte. Sans parler de la tolérance des fumeurs qui, au travail, supportent quotidiennement et sans plaintes leurs collègues qui ne fument pas.

Il va de soi que de telles clarifications linguistiques ne peuvent pas véritablement troubler une longue amitié. Mon copain ajoute même que l'emploi du terme tolérance n'est pas tout à fait approprié dans ce cas, mais que dans le fond, on comprend bien de quoi il s'agit. Et c'est vrai: le fumeur renonce temporairement à sa passion - il a de l'égard pour les personnes qui l'entourent - sans ne devoir rien tolérer de leur part. Ce que le fumeur doit en revanche bon gré mal gré supporter, c'est la pression de sa propre addiction (qui peut être importante), une petite différence, qui reste néanmoins de taille.

Quoi qu'il en soit, se dit-on au moment de sortir, jetant un dernier regard aux nombreux clients attablés, la vision quelque peu troublée par les volutes de fumée et une conjonctivite naissante; qu'il s'agisse de tolérance ou d'égards, on ne saurait qualifier, même avec la meilleure volonté du monde, le résultat final de concluant.

Bruno Kesseli 